\section{«Unis pour réussir»}

A l'occasion de mon entrée en fonction au CC, je disais dans mon éditorial de juin 2006: «Nous sommes non seulement toutes et tous concernés par les nombreux problèmes actuels de la santé, mais nous devons aussi les résoudre ensemble!»

«Unis pour réussir» est une devise qui me paraît s'appliquer tant à la FMH qu'à la vie en général: ce n'est qu'ensemble, en communiquant, que nous pouvons résoudre les problèmes qui se posent.

Ces derniers temps, diverses décisions ont été préparées par la FMH en collaboration avec les organisations faîtières (FMPP, FMCH, CMPR, SFSM), la Conférence des sociétés cantonales de médecine (CCM) et les sociétés de discipline. Bénéficiant ainsi d'un large appui interne, les messages que transmet la FMH, de même que les prises de position qu'elle publie, ont un impact marqué sur les assureurs et les politiques. Les thèses de la FMH sur l'obligation de contracter et la gestion des soins, ou les consultations concernant la carte d'assuré ou la révision de l'OAMal en sont de bons exemples.

De même, lors de la réorganisation du domaine «Tarifs et conventions», on a créé avec le «Bureau des tarifs» un instrument et des processus qui permettront à l'avenir d'élaborer et même de prendre des décisions souvent importantes, en matière tarifaire, décisions recueillant alors l'adhésion de tous les protagonistes. Ce Bureau des tarifs, qui réunit le domaine tarifaire de la FMH, les organisations faîtières et la CCM, gère, coordonne et soutient les activités liées aux tarifs. Les premières séances communes ont montré que cette instance nouvelle est bien acceptée et que ce bureau pourrait devenir une institution-clé.

«Unis pour réussir»: l'importance d'une telle coopération avait déjà été perçue à l'époque par les sociétés cantonales de médecine lorsqu'elles ont créé le G7 (l'actuelle CCM). C'est sur cette base que les conventions TARMED, le pilotage de la neutralité des coûts et le développement de la convention prestations-prix ont pu être menés à terme avec succès. Notamment grâce à l'important poids politique et à la ténacité de la CCM, on est parvenu à conclure avec santésuisse des conventions acceptables pour le corps médical, dont les points essentiels sont la parité des données, la convergence et les facteurs de prestations.

Ou encore ceci: en fondant en 2003 la région tarifaire de Suisse orientale, les sociétés cantonales concernées (AI/AR, GL, SG, SH, TG) se sont elles aussi décidées en faveur de la coopération et ont réussi à boucler favorablement la gestion de la neutralité des coûts. Toutes les tentatives de santésuisse de diviser la région à dater du $1^{\mathrm{er}}$ janvier 2007 ont échoué face à la détermination des sociétés cantonales et ce, malgré certaines offres «alléchantes» des assureurs. Les demandes de santésuisse visant à faire fixer la valeur du point par les autorités cantonales n'ont pas trouvé grâce auprès de ces dernières: les gouvernements de Suisse orientale ont prolongé la convention régionale d'adhésion et, partant, renforcé la cohésion de la région tarifaire.

Etonnamment, santésuisse a ensuite fait part, en été 2006, de sa disponibilité à négocier les valeurs du point pour 2007. Ces négociations sont maintenant près d'aboutir.

«Unis pour réussir»... Les exemples cités le montrent clairement: ce n'est qu'unis que nous parviendrons à maîtriser les grands défis du système de santé. La participation de chacun est ici importante et nous comptons aussi sur la vôtre!

Cet esprit de coopération, s'il continue d'inspirer les activités de la FMH, devrait l'aider à affermir sa position.

Dr Ernst Gähler, membre du Comité central de la FMH 